\title{
longtail1
}

\section{$5 \mathrm{~ms}$ bin ISI histograms}

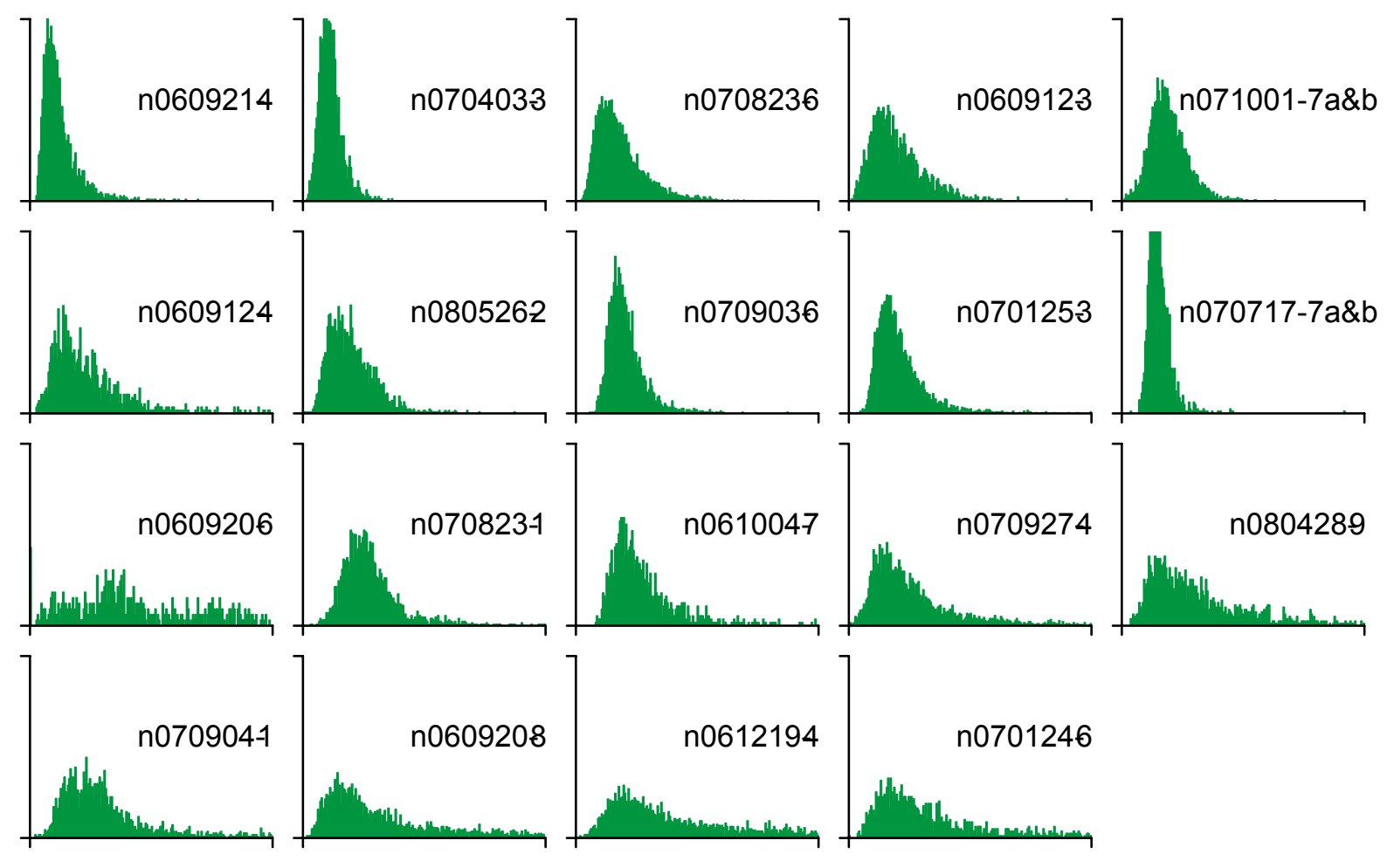

$X 0$ to 1000 (ms)

Y 0 to 500 (normalised ISIs) 


\section{longtail2}

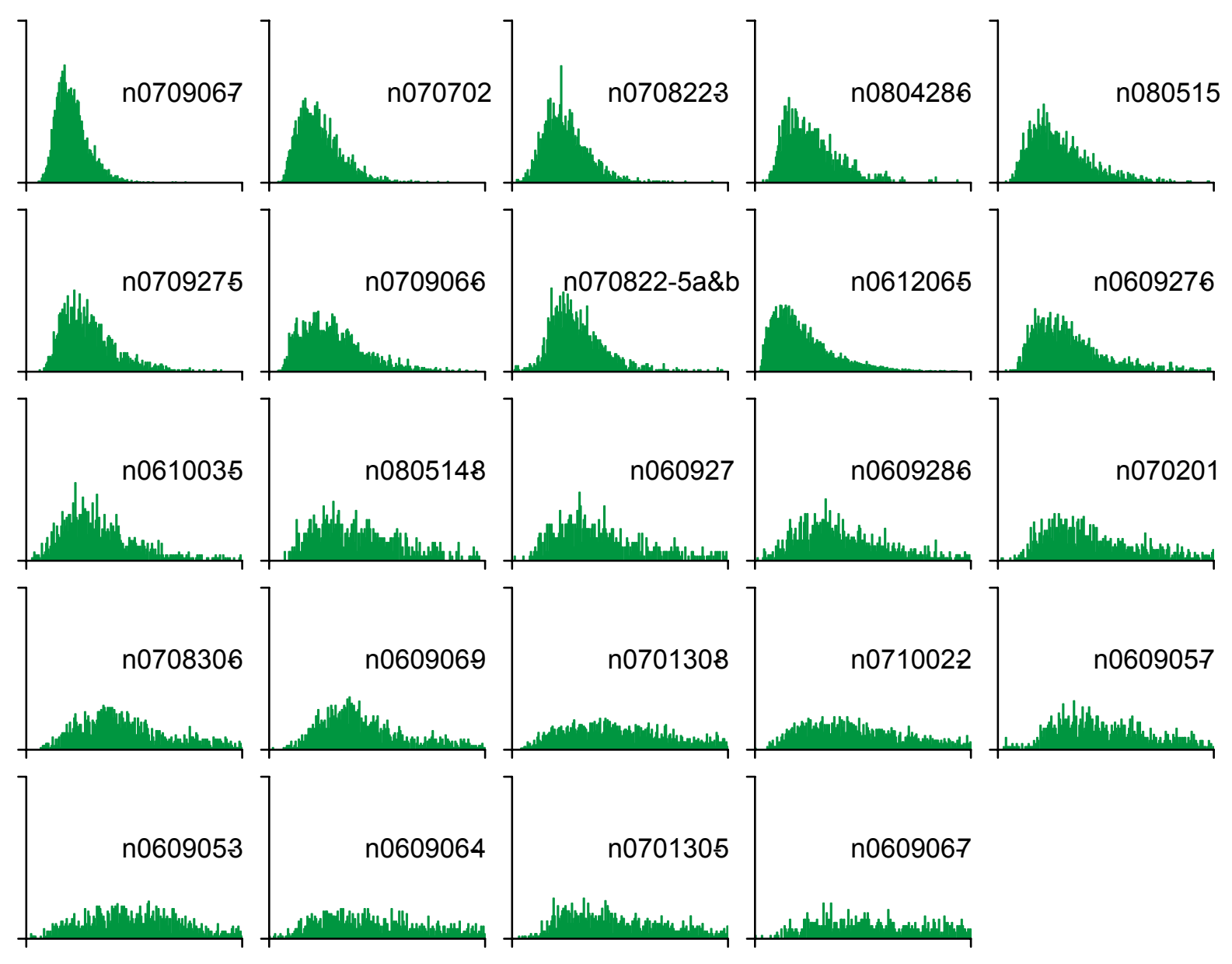




\section{broad}

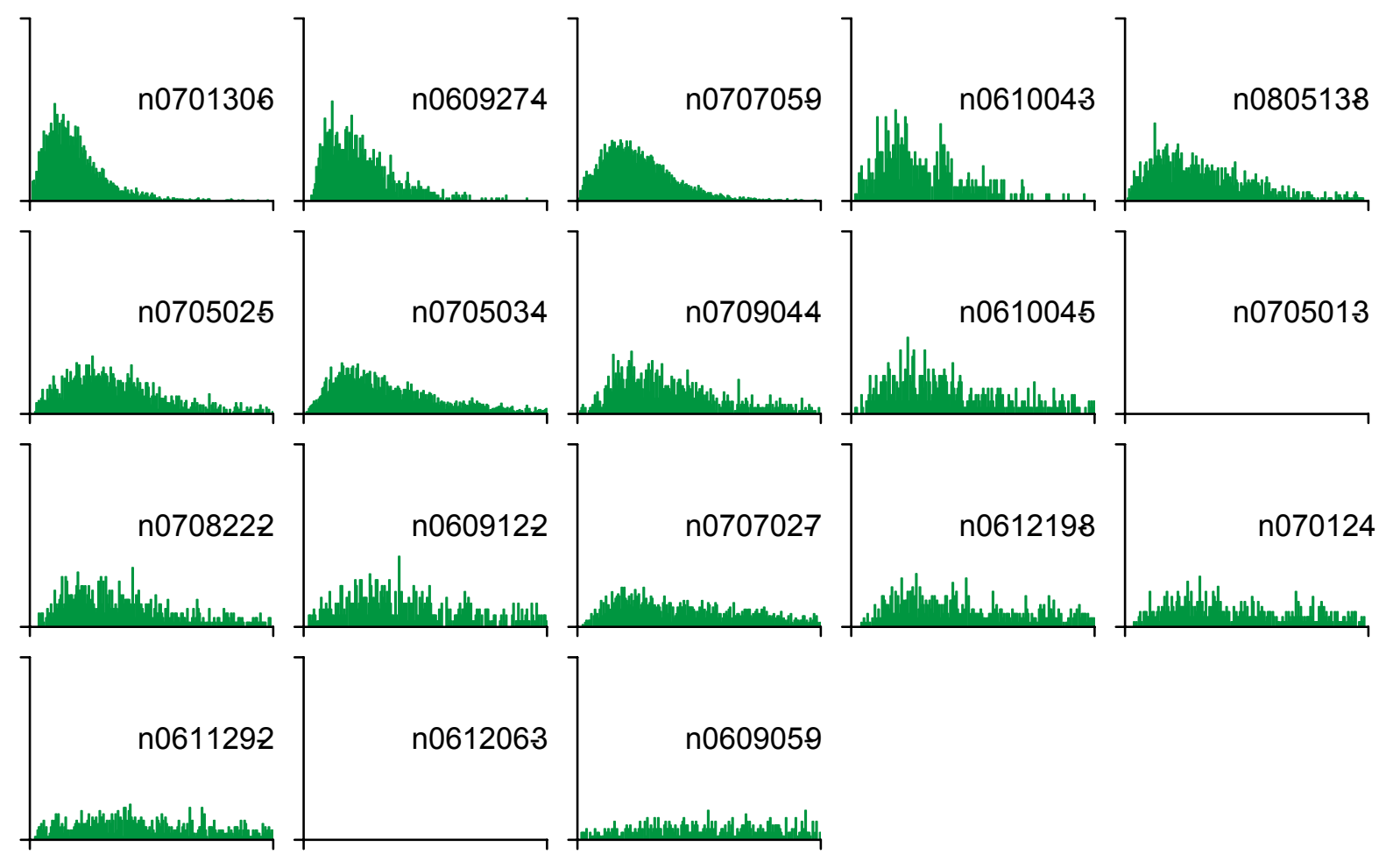




\section{doublets}

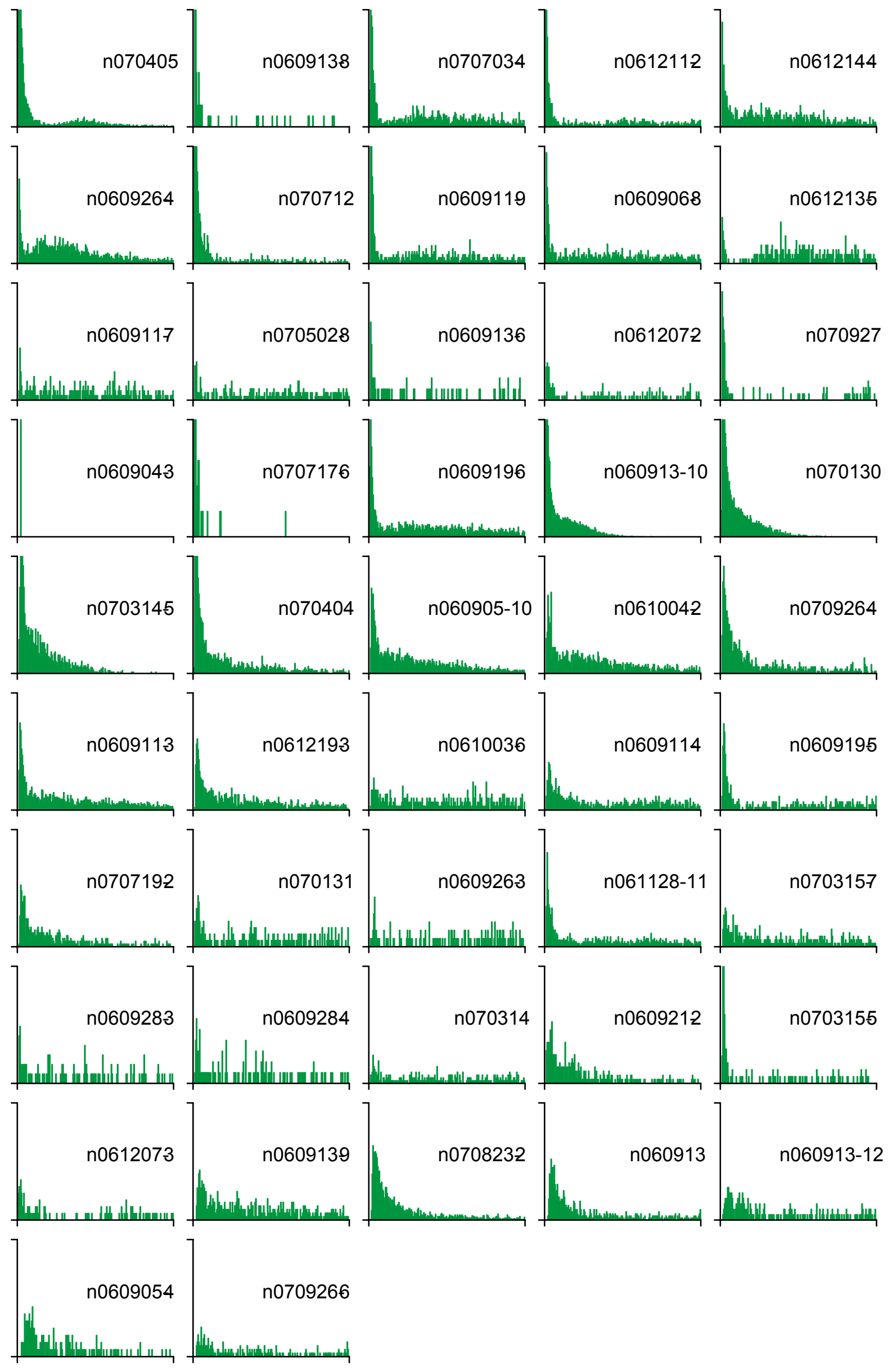




\section{doublet-broad}

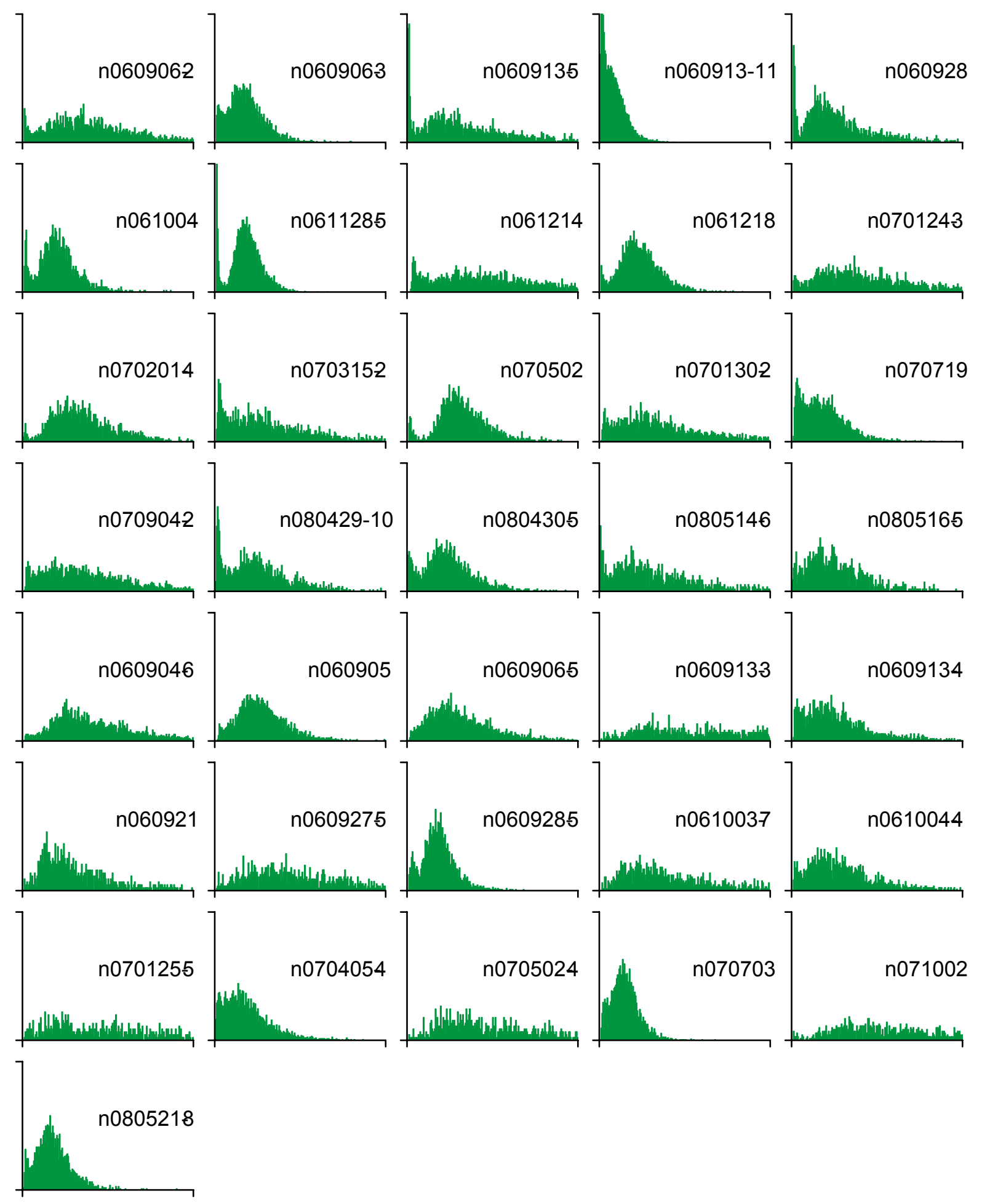




\section{oscillatory}

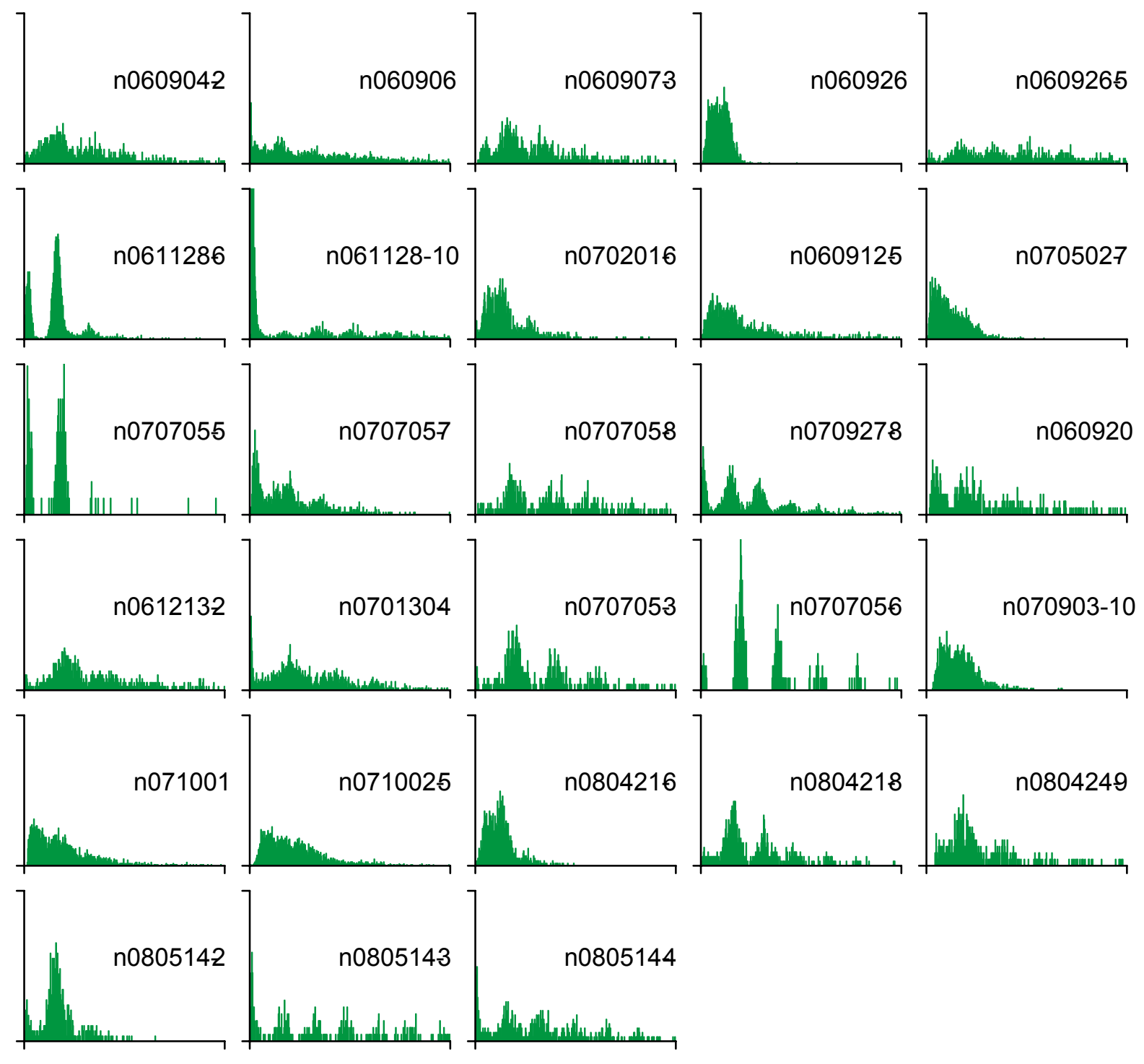

\section{$X 0$ to 2000 (ms) \\ Y 0 to 500 (normalised ISIs)}




\section{random}

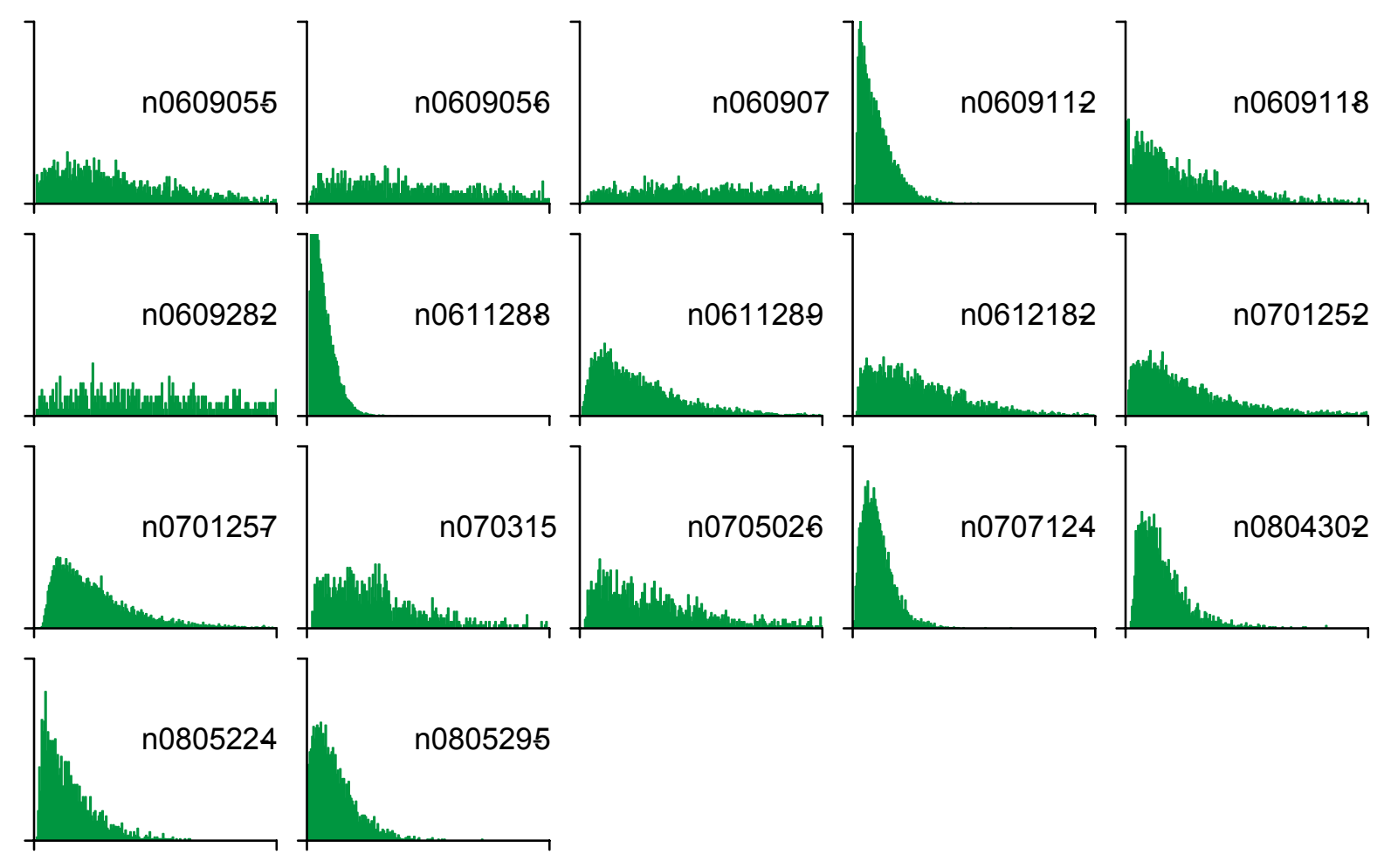




\section{slow DAP}

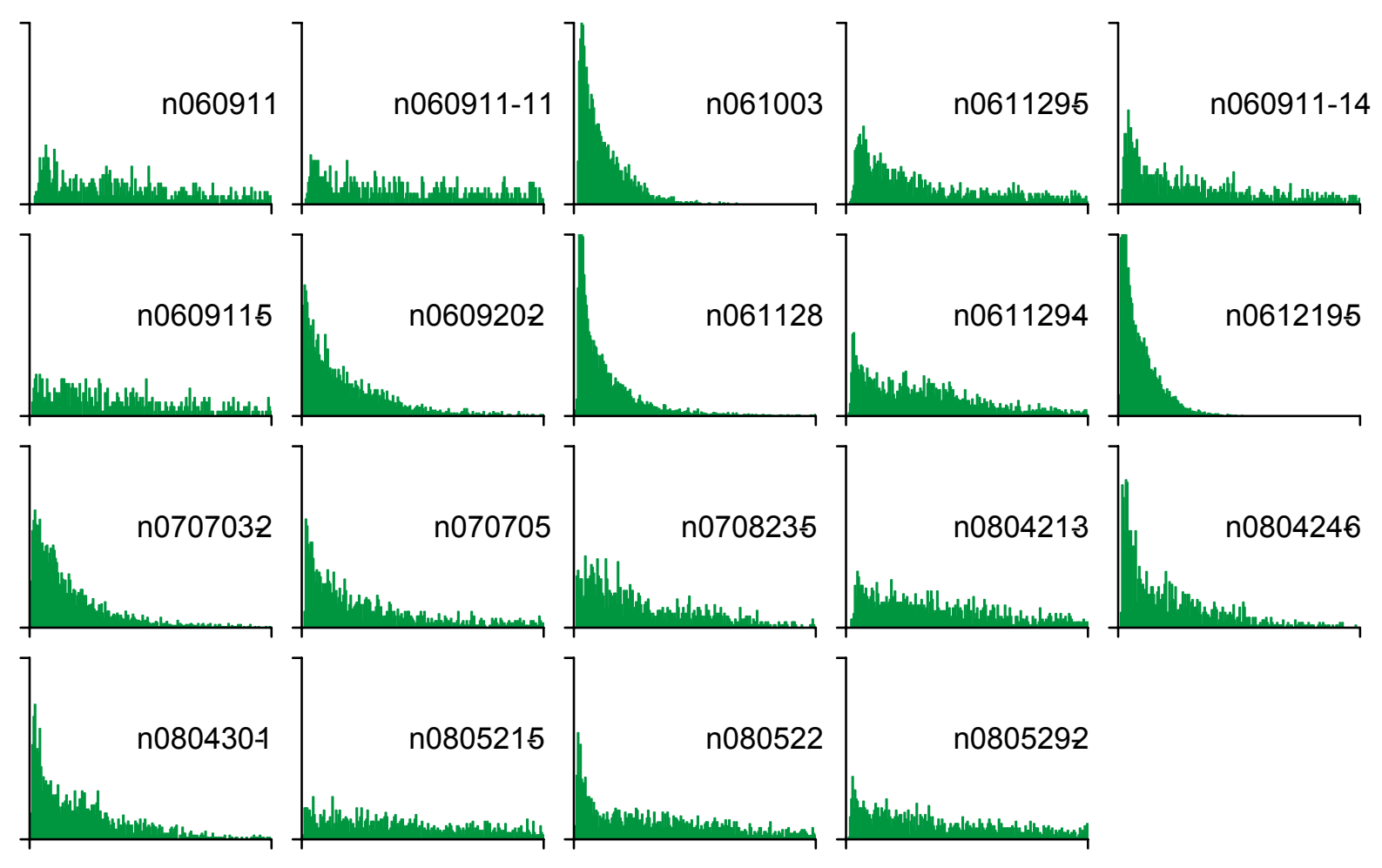




\section{regular}

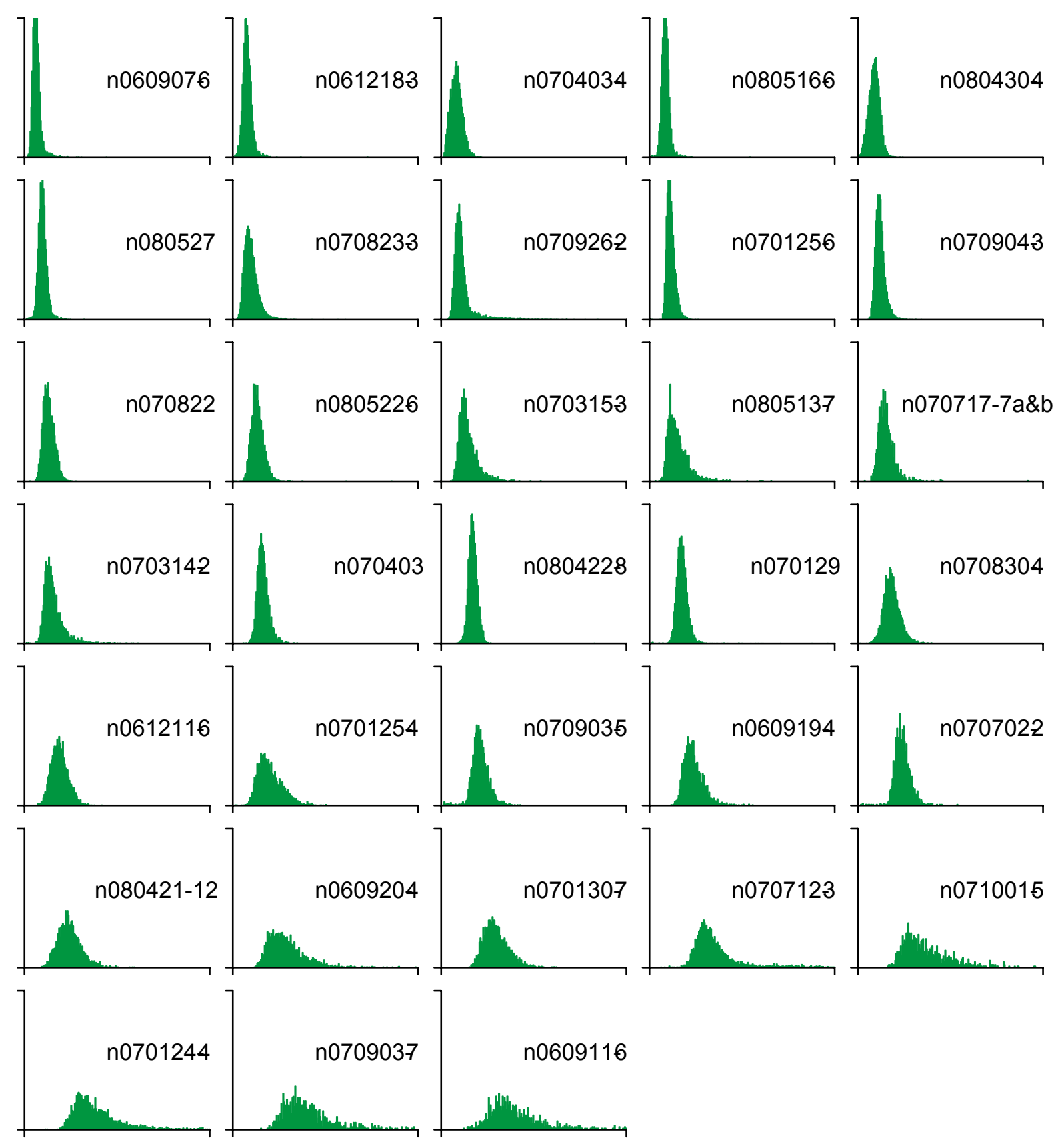

\section{$X 0$ to 1000 (ms) \\ Y 0 to 1000 (normalised ISIs)}

